\title{
A supramolecular hydrogel as a reusable heterogeneous catalyst for the direct aldol reaction $\dagger$
}

\author{
Francisco Rodríguez-Llansola, Juan F. Miravet* and Beatriu Escuder*
}

Received (in Cambridge, UK) 6th August 2009, Accepted 26th October 2009

First published as an Advance Article on the web 9th November 2009

DOI: $10.1039 /$ b916250j

An L-proline based supramolecular hydrogel is used as an efficient heterogeneous organocatalyst for the direct aldol reaction with high stereoselectivity (up to $90 \%$ ee) and recyclability (up to 3 runs). The reversible nature of this self-assembled supramolecular system allows for easy recovery and regeneration of the catalyst.

The development of catalytic systems for organic transformations in aqueous media presents an enormous interest from an environmental as well as from an economic point of view since it combines both atom economy and the greener solvent, water. ${ }^{1}$ In this field not only soluble but also heterogeneous systems have attracted the attention of researchers. For instance, different catalytic organic reactions have been carried out in aqueous media with organic and inorganic polymer supported catalysts, ${ }^{2}$ in micellar dispersions ${ }^{3}$ or in microemulsions ${ }^{4}$ among others.

A special class of phase separated organized media correspond to self-assembled fibrillar networks formed by low-molecular weight molecules, the so-called supramolecular hydrogels. ${ }^{5}$ In those, small molecules reversibly self-assemble by non-covalent interactions, being the 'hydrophobic effect' the main driving force in water. These fibrillar assemblies may present enhanced catalytic properties and product selectivity as a consequence of the appearance of cooperative interactions by proximity of catalytic groups. For example, Stupp has described fibrillar aggregates in water that are active catalysts for the hydrolysis of esters. ${ }^{6}$ Furthermore, we have recently described L-proline derivatives which are capable of selfassembly into organogels and develop catalytic activity for the Henry nitroaldol reaction only in the aggregated state as a consequence of a considerable enhancement of basicity due to proximity of L-proline groups in the gel fibers. ${ }^{7}$

Here we describe L-proline amphiphilic derivative $\mathbf{1}$ as a strong hydrogelator, and its use in a supramolecular heterogeneous catalytic system for the direct aldol reaction between cyclohexanone and 4-nitrobenzaldehyde (Scheme 1). This reaction, widely studied in organic solvents with the use of many different L-proline based catalysts, ${ }^{8}$ has been also described to take place in water and a great effort has been devoted to understand the role played by this solvent. ${ }^{9}$ In particular, Barbas has described related amphiphilic catalysts

Universitat Jaume I, Dpt. Química Inorgànica i Orgànica,

12071 Castelló,Spain.E-mail: escuder@qio.uji.es,miravet@qio.uji.es; Fax: + 34-964729155

$\dagger$ Electronic supplementary information (ESI) available: Synthesis and characterization of compounds and additional details of catalytic experiments. See DOI: 10.1039/b916250j

that form an emulsion in water and act as efficient catalysts for this reaction. ${ }^{10}$ In the case reported here, an additional advantage appears, owing to the fact that a hydrogel can be easily recycled and recovered as a solid-like heterogeneous phase. Furthermore, due to their dynamic reversible nature, supramolecular hydrogels can be disassembled in response to slight environmental changes like temperature or $\mathrm{pH}$, allowing on-off switching of catalytic activity.

Compound 1 was prepared in high yield following simple peptide coupling methodology (see ESI $\dagger$ ). This compound formed hydrogels above a concentration of $2 \mathrm{mM}$ (0.8 $\mathrm{mg} \mathrm{mL}^{-1}$ ) after dissolution in hot water and sudden cooling at $25{ }^{\circ} \mathrm{C}$ accompanied by $1 \mathrm{~min}$ of sonication. The morphology of the gel was observed by scanning electron microscopy (SEM, Fig. 1) revealing the presence of a network of ribbons of several $\mu \mathrm{m}$ length and less than $300 \mathrm{~nm}$ in width. $\mathrm{X}$-Ray powder diffraction of the xerogel was consistent with a lamellar structure in which compound $\mathbf{1}$ formed a bilayer with intercalation of the alkyl tails (Fig. 2).

Initially catalytic tests were performed by adding reagents directly on top of the hydrogel at $25{ }^{\circ} \mathrm{C}(0.16 \mathrm{mmol}$ of 4-nitrobenzaldehyde in $155 \mu \mathrm{L}$ of cyclohexanone). After $36 \mathrm{~h}$ the reaction was extracted with toluene yielding the aldol quantitatively (Table 1, entry 1). However, diastereoselectivity was moderate (anti:syn, 75:25) and enantioselectivity was poor $(12 \%$ ee). Alternatively, reagents were added on top of the hydrogel dissolved in $1 \mathrm{~mL}$ of toluene. In this case, a quantitative yield was obtained after $16 \mathrm{~h}$ at $25{ }^{\circ} \mathrm{C}$ and the toluene phase could be decanted and, after evaporation of solvents under an air current, directly analyzed by NMR and chiral-phase HPLC (entry 2, see ESI $\dagger$ ). Remarkably, the anti: syn diastereoselectivity was improved up to $91: 9$, but stereoselectivity was still quite low $(18 \%$ ee). However, when the reaction was performed at $5{ }^{\circ} \mathrm{C}$, after $24 \mathrm{~h}$ the aldol $(S, R)$-ant $i$ was obtained as the major product ( $88 \%$ ee, entry 3$)$. This is a very remarkable improvement in the selectivity considering the relatively small reduction in the reaction
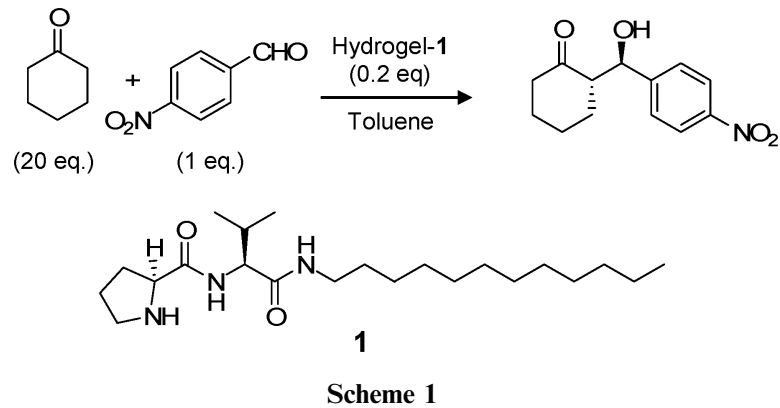


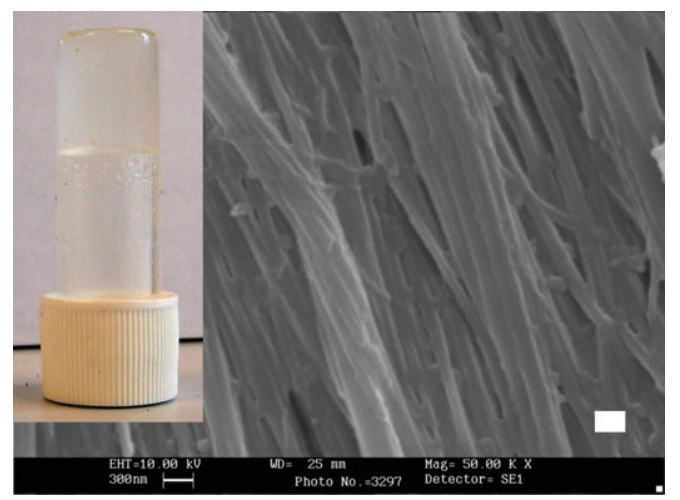

Fig. 1 Macroscopic (left) and microscopic (right) aspect of hydrogel-1; (bar: $300 \mathrm{~nm}$ ).

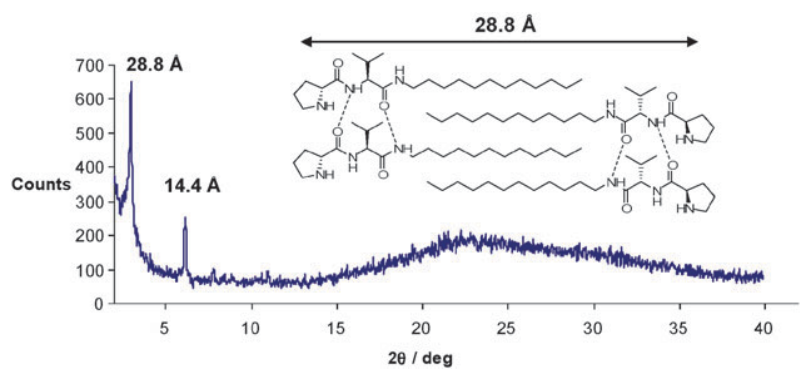

Fig. 2 X-Ray powder diffractogram of dried hydrogel-1 and a possible bilayer structure.

temperature. This behavior could be related to restricted mobility of the reactants as well as to the structural rigidification of the catalytic supramolecular aggregates. Accordingly, this would allow for a better discrimination of the reaction pathways that lead to the different stereoisomers.

Furthermore, after decantation of the organic layer, the hydrogel did not seem to be macroscopically affected and could be reused for at least two additional runs with the same efficiency and stereoselectivity (entries 4 and 5, and Fig. 3). On the other hand, the temperature and $\mathrm{pH}$ responsiveness of the hydrogel allowed for complete recovery and regeneration of the catalytic hydrogel. Thus, after use the gel was mechanically broken, washed with toluene $(2 \times 1 \mathrm{~mL})$ and formed again by heating and sonication. Alternatively, acidification of the aqueous phase would lead to protonation and disassembly, and after exhaustive washing with toluene, the system could

Table 1 Catalytic performance of hydrogel-1 ${ }^{a}$

\begin{tabular}{llllll}
\hline Entry & $T /{ }^{\circ} \mathrm{C}$ & $t / \mathrm{h}$ & Yield $^{c}(\%)$ & anti:syn & ee $^{d}(\%)$ \\
\hline $1^{b}$ & 25 & 36 & $>99$ & $75: 25$ & 12 \\
2 & 25 & 16 & $>99$ & $91: 9$ & 18 \\
3 & 5 & 24 & 98 & $92: 8$ & 88 \\
$4^{e}$ & 5 & 24 & $>99$ & $93: 7$ & 87 \\
$5^{f}$ & 5 & 24 & $>99$ & $92: 8$ & 90
\end{tabular}

${ }^{a}$ Hydrogel: $0.026 \mathrm{mmol}$ of 1 (0.2 eq.) in water $(4 \mathrm{~mL})$; Reagents: 4-nitrobenzaldehyde (1 eq.), cyclohexanone (20 eq.) in toluene (1 mL). ${ }^{b}$ Without toluene. ${ }^{c}$ Determined by NMR spectroscopy. ${ }^{d}$ Determined by chiral-phase HPLC of the anti-product. ${ }^{e}$ Entry 3, second run. ${ }^{f}$ Entry 3 , third run.

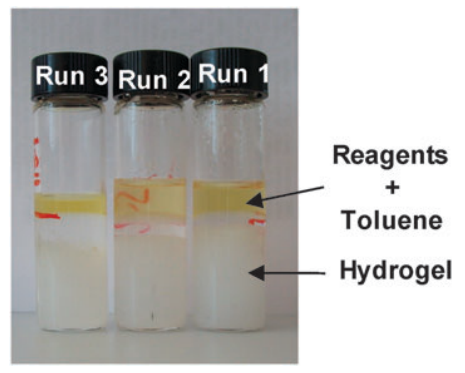

Fig. 3 Macroscopic aspect of the catalytic system used for several runs.

be switched on again by neutralization and gel formation (heating and sonication) (see ESI $\dagger$ ).

Additional experiments were performed in order to investigate two fundamental questions: (i) which was the catalytic species and (ii) where was the reaction taking place. Supramolecular gels are dynamic systems in which a given fraction of free molecules could remain in solution in equilibrium with the gel phase. Thus, before assuming that catalysis takes place in the gel phase it has to be proved that there is not a significant amount of molecules in solution or that those are not catalytically active. ${ }^{1} \mathrm{H}$ NMR spectroscopy is a convenient methodology for that purpose as it is well known that molecules in the gel phase are NMR silent, and by the addition of an internal standard the molar fraction of molecules in solution can be easily determined. ${ }^{11}$ In the current case, free molecules of 1 could not be detected in the ${ }^{1} \mathrm{H}$ NMR spectrum of the hydrogel in $\mathrm{D}_{2} \mathrm{O}$ at $30{ }^{\circ} \mathrm{C}$ (less than $0.2 \mathrm{mM}$ for $\left.500 \mathrm{MHz}{ }^{1} \mathrm{H} \mathrm{NMR}\right) \ddagger$ whereas signals of the hydrogelator could be observed after addition of TFA and destruction of the gel (Fig. 4).

The observed catalytic activity could arise from the disassembly of molecules from the hydrogel that would catalyze the reaction in the organic phase. For studying this possibility three different blank samples were prepared (see ESI $\dagger$ ): (i) blank 1, hydrogel + toluene; (ii) blank 2, hydrogel + toluene + cyclohexanone; and (iii) blank 3, hydrogel + toluene +4 -nitrobenzaldehyde. All the samples were equilibrated at $5{ }^{\circ} \mathrm{C}$ over 3 days and then analyzed by NMR spectroscopy revealing that none of compound $\mathbf{1}$ was

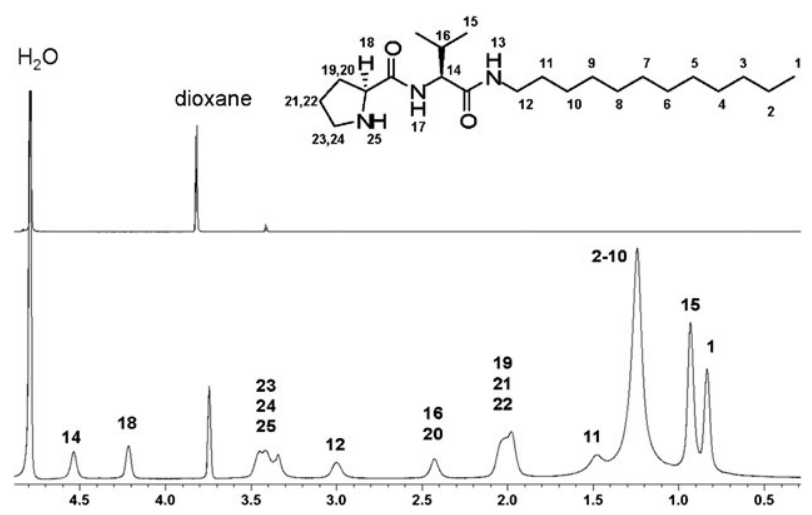

Fig. $4{ }^{1} \mathrm{H}-\mathrm{NMR}$ spectra of $\mathbf{1}$ in $\mathrm{D}_{2} \mathrm{O}([\mathbf{1}]=20 \mathrm{mM}$, dioxane was used as an internal standard $(c=2 \mathrm{mM})$ ). Top: Hydrogel; bottom: solution obtained after addition of $50 \mu \mathrm{L}$ of trifluoroacetic acid. 
present in the organic phase. Furthermore, addition of the remaining reagents to the organic phase isolated from each blank (for example, cyclohexanone and 4-nitrobenzaldehyde in blank 1) did not lead to any product after $24 \mathrm{~h}$ at $5{ }^{\circ} \mathrm{C}$, confirming that there were no catalytic species in the organic phase and demonstrating that reaction is taking place in the heterogeneous gel phase.

In conclusion, the inclusion of L-proline into a low molecular weight gelator leads to a supramolecular hydrogel with a remarkable efficiency in a benchmark direct aldol reaction. Most likely toluene (and also cyclohexanone) is helping in the transport of 4-nitrobenzaldehyde, poorly soluble in water, to the hydrophobic regions of the gel fibers. The results described indicate that this supramolecular heterogeneous catalytic system uses one of the most important non-covalent interactions in the biological context, the hydrophobic effect, both for the self-construction of the catalyst as well as for the approach of the substrate to the catalytic center. As reported for direct aldol reactions catalyzed by L-proline derivatives, ${ }^{8-10}$ the reaction is meant to take place following an enamine-based mechanism, and accordingly, the supramolecular hydrogel would be acting as an effective type I aldolase mimetic. Further work will be carried out in the study of the scope of substrate selectivity and the influence of co-solvent on the reaction rate. Finally, a straightforward methodology has been applied for recycling and regeneration of the catalytic system allowing for the complete recovery of catalytic performance.

We thank MEC (Grant CTQ2006-14984) and Universitat Jaume I-Bancaixa (Grant P1.1A2006-1) for financial support. F. R. Ll. thanks Generalitat Valenciana for a FPI fellowship.

\section{Notes and references}

$\ddagger$ The absence of catalytic activity in solution at $5{ }^{\circ} \mathrm{C}$ has been confirmed by running reactions on samples where the hydrogel has been filtered off.

1 (a) B. M. Trost, Science, 1991, 254, 1471; (b) B. M. Trost, Angew. Chem., Int. Ed. Engl., 1995, 34, 259; (c) U. M. Lindström, Chem. Rev., 2002, 102, 2751; (d) C. J. Li and L. Chen, Chem. Soc. Rev., 2006, 35, 68

2 (a) S. Minakata and M. Komatsu, Chem. Rev., 2009, 109, 711 and references cited therein; $(b)$ A. F. Trindade, P. M. P. Gois and C. A. M. Afonso, Chem. Rev., 2009, 109, 418.

3 Reactions and Synthesis in Surfactant Systems, in Surfactant Science Series, ed. J. Texter, Marcel Dekker, New York, 2001, vol. 100.

4 K. Holmberg, Eur. J. Org. Chem., 2007, 731.

5 (a) Molecular Gels: Materials with Self-assembled Fibrillar Networks, ed. P. Terech, R. G. Weiss, Springer, Dordrecht, 2006;

(b) L. Estroff and A. D. Hamilton, Chem. Rev., 2004, 104, 1201; (c) N. M. Sangeetha and U. Maitra, Chem. Soc. Rev., 2005, 34, 821.

6 M. O. Guler and S. I. Stupp, J. Am. Chem. Soc., 2007, 129, 12082.

7 (a) F. Rodríguez-Llansola, J. F. Miravet and B. Escuder, Chem. Commun., 2009, 209; (b) F. Rodríguez-Llansola, J. F. Miravet and B. Escuder, Org. Biomol. Chem., 2009, 7, 3091; (c) F. RodríguezLlansola, B. Escuder and J. F. Miravet, J. Am. Chem. Soc., 2009, 131, 11478.

8 (a) B. List, R. A. Lerner and C. F. Barbas III, J. Am. Chem. Soc., 2000, 122, 2395; (b) W. Notz, F. Tanaka and C. F. Barbas III, Acc. Chem. Res., 2004, 37, 580; (c) B. List, Acc. Chem. Res., 2004, 37 548; (d) B. List, in Modern Aldol Reactions, ed. R. Mahrwald, Wiley-VCH, Weinheim, 2004, vol. 1, pp. 161-200.

9 (a) A. P. Brogan, T. J. Dickerson and K. D. Janda, Angew. Chem. Int. Ed., 2006, 45, 8100; (b) Y. Hayashi, Angew. Chem., Int. Ed., 2006, 45, 8103; (c) C. F. Barbas III, Angew. Chem., Int. Ed., 2008, 47, 42; (d) J. Mlynarski and J. Paradowska, Chem. Soc. Rev., 2008, 37, 1502; (e) J. Paradowska, M. Stodulski and J. Mlynarski, Angew. Chem., Int. Ed., 2009, 48, 4288.

10 N. Mase, Y. Yakai, N. Ohara, H. Yoda, K. Takabe, F. Tanaka and C. F. Barbas III, J. Am. Chem. Soc., 2006, 128, 734.

11 (a) B. Escuder, M. Llusar and J. F. Miravet, J. Org. Chem., 2006, 71, 7747; (b) A. R. Hirst, I. A. Coates, T. R. Boucheteau, J. F. Miravet, B. Escuder, V. Castelletto, I. W. Hamley and D. K. Smith, J. Am. Chem. Soc., 2008, 130, 9113. 